\title{
Lauter weise Sprüche
}

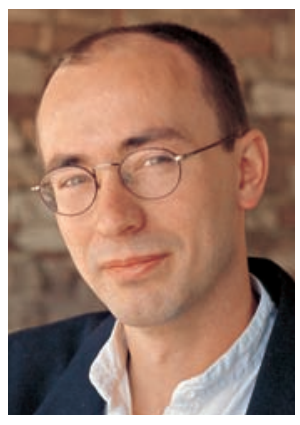

Dr. med. Dieter Schmid, Redaktionsleitung
Mediziner haben ein Talent dafür, Weisheiten in einfache Merksätze zu verpacken. „Häufiges ist häufig! “ ist so ein Spruch. Das klingt simpel und ergibt doch Sinn: Das ständige Lernen auf MC-Prüfungen im Studium führt zu einer verschobenen Wahrnehmung. Orientiert man sich nur an IMPP-Fragen, glaubt man eben irgendwann tatsächlich, dass Myotonia Curschmann-Steinert und Co. eine zentrale Rolle in der Medizin spielen. Um einen Kontrapunkt zu setzen, haben wir für diese Via medici die Herzinsuffizienz als Titelthema gewählt. Die ist nämlich tatsächlich häufig. In Europa leiden daran etwa 10 Millionen Menschen! Auf S. 46 geben wir Ihnen eine Übersicht über aktuelle und künftige Behandlungsverfahren.

Eine weitere Mediziner-Weisheit lautet: „Kinder sind keine kleinen Erwachsenen.“ Dieser Merksatz macht auf das Problem aufmerksam, dass Kindern häufig Medikamente gegeben werden, die für ihre Altersgruppe nie klinisch getestet wurden. Die Dosen werden dann einfach relativ zum Gewichtsanteil eines Erwachsenen vermindert. Das ist oft wenig sinnvoll. Schließlich verarbeiten die Kleinen die Wirkstoffe ganz anders. Der European Resuscitation Council (ERC) hat deshalb spezielle Empfehlungen entwickelt, wie Kinder im Fall eines Kreislaufstillstands reanimiert werden müssen. In unserer Praxisanleitung „Kleine Leben am seidenen Faden“ auf S. 32 erfahren Sie, wie diese Vorgaben aussehen.

Nicht medizinisch, sondern arabisch ist der Spruch: „Vertraue auf Gott, aber binde zuerst dein Kamel fest.“ Ein guter Tipp. Viele junge Mediziner vertrauen hierzulande darauf, dass sich die Arbeitssituation langfristig bessert. Trotzdem hat es Sinn, nach „Hintertürchen“ Ausschau zu halten. Die Welt ist groß, und Ärzte werden überall gebraucht - zum Beispiel auch in der Region, aus der dieser Spruch stammt! Für Mediziner, die kein Problem mit hohen Temperaturen haben, eröffnen sich dort attraktive Jobchancen. Mehr darüber in „Medizin am Golf“ auf S. 8. Und noch ein arabischer Spruch: „Ein goldener Sattel macht einen Esel noch nicht zum Pferd." Niemand würde unsere Unis als Esel bezeichnen. Aber was ist eigentlich mit den 65 Millionen Euro passiert, die Medizinstudenten letztes Jahr an Studiengebühren bezahlt haben? Hat sich die Lehre deutlich verbessert? Ein Resümee finden Sie in unserem Artikel „Fix gemacht, schlecht durchdacht“ auf S. 20.

Ich wünsche Ihnen einen guten Start ins Wintersemester! Binden Sie Ihr Kamel fest!

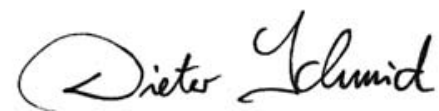

\section{Vorlesungen - reine Zeitverschwendung?}

Wer häufig in Vorlesungen geht, kommt um sie nicht herum: stickige Hörsäle mit mieser Akustik und langweilige Dozenten, die viel zu schnell die Folien wechseln. Nicht wenige Studenten ziehen daraus ihre Konsequenzen. Sie meiden Frontalvorträge aus Prinzip und beschränken sich bei der Wissensbeschaffung auf Lehrbücher, Podcasts, e-books und Internet. Andere schwören - trotz harter Holzbänke - auf den didaktischen Wert dieser Veranstaltungen.
Sie wollen auf Falldemos, Prüfungstipps und den Austausch mit Professoren und Kommilitonen nicht verzichten. Wo stehen Sie? Besuchen Sie gern Vorlesungen oder gehören Sie zu den Studenten, denen es vor Hörsaalbesuchen graust?

\section{- Glauben Sie, dass man heutzutage auf Vorlesungen verzichten kann?}

Stimmen Sie ab und sagen Sie Ihre Meinung unter: www.thieme.de/viamedici/aktuelles| aktion/miniumfrage4_08.html
- Ergebnis der Umfrage in Via medici 3.08: Wir fragten, ob Sie es unfair finden, wenn sich Kommilitonen mit Pillen für Prüfungen fit machen. Überraschung: 57\% der Teilnehmer stört das nicht, nur $36 \%$ sahen im „Lern-Doping“ ein unfaires Verhalten. Ein „Doping-Gegner“ kommentierte: „Gegen Nervosität in Prüfungen hilft gute Vorbereitung am besten!“ Ein Befürworter hält dagegen: „Ich nehme mittlerweile auch Benzos. Früher nahm ich nix und fiel überall durch. Jetzt ist das kein Problem mehr." Mehr Kommentare unter: .../aktuelles/aktion/miniumfrage3_07.html 